\title{
Role of Branched-chain Amino Acid Metabolism in Tumor Development and Progression
}

\author{
Min Kyu Jung ${ }^{1, *}$, Akinkunmi Paul Okekunle ${ }^{2,3, *}$, Jung Eun Lee ${ }^{2,3}$, Mi Kyung Sung ${ }^{4}$, Yun Jeong Lim ${ }^{5}$ \\ ${ }^{1}$ Division of Gastroenterology and Hepatology, Department of Internal Medicine, Kyungpook National University Hospital, \\ Daegu, ${ }^{2}$ Department of Food and Nutrition, College of Human Ecology, Seoul National University, ${ }^{3}$ Research Institute \\ of Human Ecology, Seoul National University, ${ }^{4}$ Department of Food and Nutrition, Sookmyung Women's University, \\ Seoul, ${ }^{5}$ Department of Internal Medicine, Dongguk University Ilsan Hospital, Dongguk University College of Medicine, \\ Goyang, Korea
}

\begin{abstract}
Branched-chain amino acids (BCAAs), isoleucine, leucine and valine, are essential amino acids with vital roles in protein synthesis and energy production. We reviewed the fundamentals of BCAA metabolism in advanced cancer patients. BCAAs and various catabolic products act as signalling molecules, which activate mechanisms ranging from protein synthesis to insulin secretion. Recently, BCAA metabolism has been suggested to contribute to cancer progression. Of particular interest is the modulation of the mTOR activity by BCAAs. There are likely multiple pathways involved in BCAA metabolism implicated in carcinogenesis. Understanding the mechanism(s) underlying altered BCAAs metabolism will significantly advance the current understanding of nutrient involvement in carcinogenesis and direct future studies to unravel the significance of BCCA metabolites in tumor development and progression.
\end{abstract}

Key Words Amino acids, branched-chain, Isoleucine, Leucine, Valine, Neoplasm

\section{INTRODUCTION}

Tumor cells often potentially undergo uncontrolled and unlimited evolution which is dependent on the availability of some metabolites in the context of tumor microcosm for survival $[1,2]$. Several nutrients [3-5] have been documented to promote tumor growth through complex metabolic pathways that are modulated by environmental factors, lifestyle changes, genetic predispositions, cellular interactions, and metabolic manipulations [6-8].

The significance of branched-chain amino acids (BCAAs) has been documented in multiple chronic diseases [9-13]. Whether the pathophysiology of BCAA metabolism in chronic diseases is potentially associated with cancer biology is yet to be clearly understood. The vitality of such association cannot be underestimated in proposing novel strategies for tumor management and treatment to improve the quality of life and wellbeing of populations. This short review summarizes role for BCAA metabolism in tumor development and progression.

\section{THE BIOCHEMISTRY OF BCAAS}

BCAAs including isoleucine, leucine and valine (Fig. 1) are essential amino acids synthesized in low quantities, and they constitute approximately $35 \%$ of all essential amino acids [14]. BCAAs regulate protein metabolism via multiple pathways, primarily those involving the mTOR [15]. They are the mostly hydrophobic amino acids with a vital role in proteins' structural and membrane integrity [16]. BCAAs have unique similarities that are most often studied together (dietary consumption, combustion, and metabolism). However, they differ significantly in terms of morphology, hydrophobicity and biological effects [17].

BCAAs are abundantly synthesized in bacteria, plants, and fungi but not in animals [18]. Similar enzymes synthesize valine and isoleucine, but leucine is usually produced from $\alpha$-ketoisovaleric acid, a transamination precursor of valine [19]. Also, carbon elements in valine and leucine are derived from pyruvate, but isoleucine carbons are derived

Received September 13, 2021, Revised September 30, 2021, Accepted November 8, 2021

Correspondence to Yun Jeong Lim, E-mail: drlimyj@gmail.com, https://orcid.org/0000-0002-3279-332X

Check for updates

*These authors contributed equally and are joint first authors for this work. 
<smiles>CC(C)C[C@H](N)C(=O)O</smiles><smiles>CC[C@H](C)[C@H](N)C(=O)O</smiles>

from the relatively rare threonine, reflecting the conserved protein abundance ratio [18]. Similarly, valine and isoleucine are more rampant in $\beta$-sheets, but leucine features more in the $\alpha$-helices [20]. In mammals, BCAAs are primarily catabolized in the hepatic tissues or skeletal muscles [21-23] by branched-chain aminotransferases (BCATs) to branchedchain $\alpha$-keto-acids (BCKAs) [24].

\section{THE PHYSIOLOGY OF BCAAS}

Under normal conditions, there is a precise balance between the intake and expenditure of BCAAs [22]. Diet is likely the only significant source of BCAAs, even though gut microbiota could synthesize BCAAs in relatively minimal and insignificant amounts [25]. Most BCAAs are lost through oxidative catabolism in the body, but the amount of BCAAs in urine is usually negligible to juxtapose the loss. Average circulating levels of BCAAs in the fasting state, i.e. approximately 200 $\mu \mathrm{M}$ of valine, $100 \mu \mathrm{M}$ of leucine, and $60 \mu \mathrm{M}$ of isoleucine [23], are usually well maintained within hours after feeding [2628]. BCAAs (derived from the diet or released from protein breakdown that appears in the circulation) are released into tissues where they can be oxidized or incorporated into newly synthesized proteins $[23,29,30]$.

BCAA intake and utilization are likely to vary depending on age and sex differences of individuals as observed in protein intake. The usual daily minimum requirement of protein intake to maintain muscle mass is $0.8 \mathrm{~g} / \mathrm{kg} / \mathrm{day}$, though recent recommendations for a healthy diet are higher [31]. The average protein intake among males is $1.7 \mathrm{~g} / \mathrm{kg} /$ day, which is equivalent to 88,145 , and $66 \mathrm{mg} / \mathrm{kg} /$ day intakes of valine, leucine, and isoleucine, respectively. However, protein intake is lower among females and declines with age [32].

Most of the BCAAs (Fig. 2) in the circulatory pool are reincorporated into newly synthesized proteins, typically accounting for $7,090 \%$ of disposal in the fasting state [33-35]. Protein synthesis requires an anabolic signal for amino acid assembly into new proteins [23]. Importantly, leucine plays the role in an anabolic signal transduction [36]. In skeletal muscles, oral administration of leucine (but not isoleucine or valine) stimulates protein synthesis $[37,38]$. Many of these effects are likely to be initiated by hormonal (e.g., insulin or insulin-like growth factor [IGF]-I) and amino acid signals primarily mediated by mTOR [39]. Hence, whole-body BCAA metabolism reflects a balance among the complex network of protein intake, protein synthesis cycle, breakdown and oxida-<smiles>CCC(C)[C@H](N)C(=O)O</smiles>

Figure 1. Chemical structures of branched-chain amino acids.

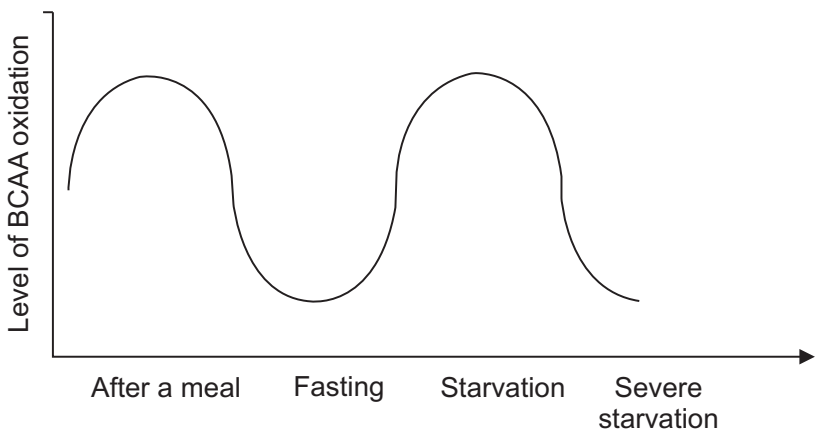

Figure 2. Relationship between BCAAs oxidation and diet. Intracellular BCAA oxidation is dependent on a constellation process manipulated by several states of dietary availability; after a meal, fasting, starvation and severe starvation. BCAAs oxidation increases after a meal but falls in a fasting state. BCAA oxidation increases in the liver during starvation and is primarily driven by gluconeogenic precursors in the TCA cycle. In severe starvation, BCAAs oxidation falls, likely to preserve essential amino acids for other metabolic functions critical for functional survival in the cytosol. BCAA, branchedchain amino acid; TCA, tricarboxylic acid.

tion of BCAAs, etc.

\section{THE PATHOPHYSIOLOGY OF BCAA METABOLISM IN MULTIPLE CANCERS}

Since BCAAs are essential amino acids, they are also utilized by cancer cells: derived either from circulation or surrounding tissues. Alterations in circulating BCAAs levels in patients diagnosed with cancer have long been noted in observational studies [40-43]. A retrospective metabolomic study demonstrated that elevated plasma BCAA levels are associated with more than a two-fold increase in the risk of pancreatic cancer independent of the intermediate development of diabetes [40]. Similarly, mice bearing Kras-driven pancreatic tumors displayed higher plasma levels of BCAAs before the manifestation of subclinical cancers [40]. Interestingly, the same appears not true for other tumors, even when driven by the same mutations in Kras [44]. It remains unclear whether these alterations in systemic BCAAs metabolism contribute to tumor growth or metastasis.

Leucine is a well-described mTOR agonist [45]. In addition, Sestrin2 was identified as a direct intracellular leucine sensor and an mTOR complex 1 (mTORC1) regulator [46,47]. Many studies on BCAA metabolism in tumor manifestations have focused on BCAT1. The expression of BCAT1 is altered in 
numerous cancers (Fig. 3) and correlates with poor tumor outcomes [48-51]. BCAT1 expression in glioblastoma, an aggressive type of cancer that can occur in the brain or spinal cord, is specific to those carrying wild-type isocitrate dehydrogenase 1 (IDH1) and 2 (IDH2) [52]. Mutations in either IDH1 or IDH2 can suppress BCAT1 through DNA methylation and epigenetic silencing [52]. By means of depleting $\alpha$-ketoglutarate, the mutation in IDH generates the oncometabolite, 2-hydroxyglutarate that potently inhibits $\alpha$-ketoglutarate-dependent dioxygenases, including histone demethylases [53] and ultimately promotes growth of cancer (stem) cells via hypoxia-inducible factor- $1 \alpha$ stabilization and by altering the epigenetic mechanism [54]. Conversely, 2-hydroxyglutarate can limit the supply of glutamate by inhibiting 2-oxogluta-

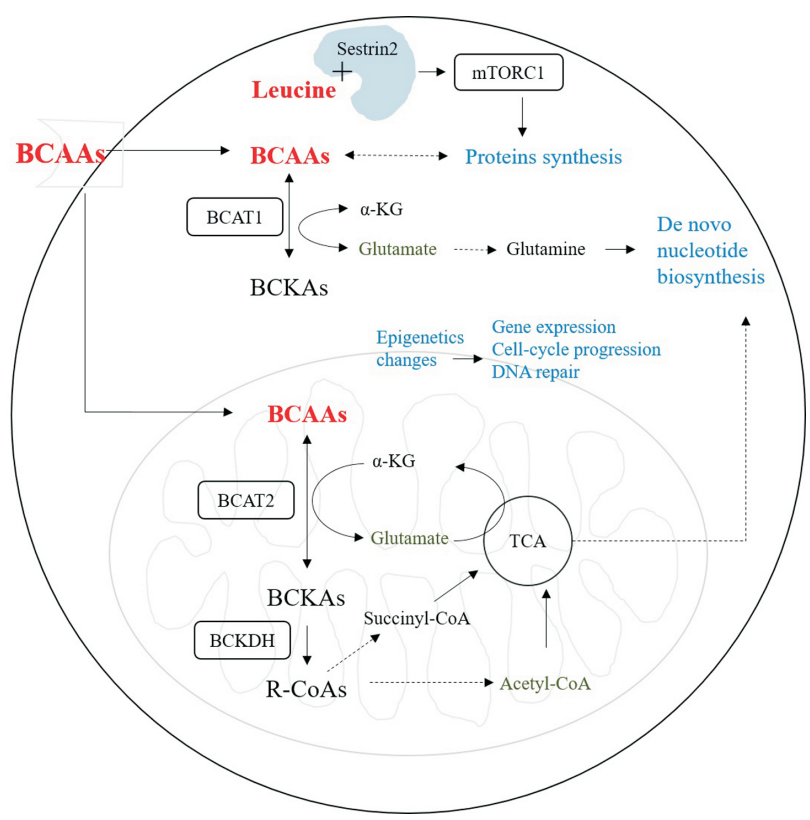

Figure 3. A model of BCAA metabolism in tumor manifestation. Cancer cells are likely to obtain BCAAs from the tumor microenvironment or protein degradation as essential amino acids. BCAAs play distinct roles in cancer cells. Thus, BCAAs can activate the mTORC1 signalling, which stimulates protein translation, growth, and survival. They also serve as building blocks in protein synthesis and can be metabolized into BCKAs in the cytosol by BCAT1 and/ or mitochondria by BCAT2, a process involving the conversion of $\alpha-K G$ to glutamate. BCAAs are also used as indirect nitrogen sources for nucleotide and non-essential amino acid biosynthesis via the glutamate-glutamine axis, and further catabolized to yield acetyl-CoA and succinyl-CoA that feed into the TCA cycle, thereby contributing to energy production. The acetyl-CoA levels have an impact on the epigenetic changes of cells. It can influence diverse cellular processes, such as gene expression, cell-cycle progression and DNA repair. In some cancers such as chronic myeloid leukemia, BCAT1 is thought to convert BCKAs back to BCAAs. mTORC1, mTOR complex 1; BCAAs, branched-chain amino acids; BCAT, branched-chain aminotransferase; $\alpha$-KG, $\alpha$-ketoglutarate; BCKAs, branched-chain $\alpha$-keto acids; TCA, tricarboxylic acid; $\mathrm{BCKDH}$, branched-chain $\alpha$-keto acid dehydrogenase; R-CoAs, branched-chain acyl-CoAs. rate-dependent transaminases such as BCAT1 and BCAT2, thereby promoting dependence on glutaminase for glutamate biosynthesis [55] and conferring vulnerability to repression of cancer cell proliferation by glutaminase inhibitors [56]. Also, BCAT1 overexpression can promote mTORC1 activity in breast cancer through complex mechanisms [57].

Mammalian lethal giant larvae homolog 2 (LLGL2) is a scaffolding protein that plays a role in regulating the mobilization of apical-basal polarity in epithelial cells and solute carrier family 7 member 5 (SLC7A5) [58]. LLGL2 has been implicated in leucine uptake and proliferation of estrogen-sensitive breast cancer cells under excessive leucine stress [59]. However, data from epidemiologic studies alluding to the significance of dietary and plasma BCAAs in breast cancer manifestation are divergent $[43,60]$.

Compared to healthy controls, a negative differential fold difference of plasma valine and leucine has been reported among colorectal cancer (CRC) patients in China [61]. Similarly, plasma leucine and valine concentrations were inversely related to odds of CRC among Japanese adults, particularly males [42]. In another study among CRC patients, BCAAs were unrelated to $\mathrm{CRC}$-specific survival, but 2-ethylhydracrylic acid, a BCAA metabolite, was significantly associated with reduced CRC-related mortality [62]. Likewise, another BCAA metabolite, leucyl-leucine was inversely associated with CRC occurrence in a study on metabolomic profiling in the United States [63]. The mechanisms responsible for this association are yet to be clearly understood, but manipulating the IGF-I expression might be a plausible route. First, IGF-I can promote colon carcinogenesis by facilitating the formation of premalignant lesions such as $\beta$-catenin accumulated crypt and aberrant crypt foci [64-66]. However, lower expression of IGF-I has been reported in BCAA-supplemented C57BL/KsJ$D B / d b$ mice treated with azoxymethane [67]. Second, BCAA turnover was higher in primary colon carcinoma tissues than normal colon mucosa [68]. Third, an ex vivo/in vitro report [69] demonstrated that isoleucine, in a mouse liver metastatic colon cancer model, partly exerted antiangiogenic effects via the mTOR pathway by inhibiting (VEGF) synthesis. In line with these observations, two recent epidemiological reports from Italy [70] and the United States [71] found a modest inverse relationship between dietary BCAA intake and CRC. In another study, branched-chain $\alpha$-keto acid dehydrogenase $(\mathrm{BCKDH})$ expression has been linked to CRC tumorigenesis via the mitogen-activated protein kinase (MAPK) pathway ex vivo [72].

Some reports have itemized the role of BCAA in pancreatic cancer. For example, elevated BCAA uptake was related to progression of pancreatic ductal adenocarcinoma (PDAC) [73]. Also, BCKDH knockdown inhibited lipogenesis and reduced the proliferation of PDAC cells. Similarly, the elevated levels of circulating BCAAs are considered an early event in human pancreatic adenocarcinoma development [40]. Furthermore, evidence for the autochthonous (and not Kras-driv- 
en) tumor manifestation localized to the pancreas associated with elevated BCAA has been reported [40]. These results consistently suggest that BCAAs are associated with PDAC.

In chronic myelogenous leukemia (CML) blast crisis, BCAT1 overexpression resulted in increased intracellular concentrations of BCAAs by aminating branched-chain $\alpha$-keto acids (BCKAs) to BCAAs [74]. In this study, inhibition of BCAT1 expression reduced mTORC1 activity, presumably by reducing intracellular BCAA concentrations. Importantly, from a therapeutic standpoint, BCAT1 knockdown in a CML mouse model improved survival, while the use of the BCAT1 inhibitor gabapentin suppressed colony formation of CML in human patients [74]. The most well known epigenetic mechanism of BCAT1 expression is the mutation of IDH. Another epigenetic mechanism involving the disruptor of telomeric silencing 1-like (DOT1L) histone methyltransferase was proposed by Oktyabri and colleagues [75]. DOT1L activates BCAT1 gene expression through histone $\mathrm{H} 3$ lysine79 methylation of the coding region [75]. In leukemias driven by genetic mutation of the mixed-lineage leukemia 1 (MLL1) gene, DOT1L maintains an open chromatin state and gene transcription [75].

The cancer-specific expression of BCAT1 is likely to be a promising target for therapeutic interventions in cancer treatment. However, the biological functions of BCAT1 in cancer are not well understood, but may be dependent on the cancer tissue of origin $[44,76]$. Accumulating evidence supports the vital and multifaceted role of BCAT1 in the development and progression of multiple types of cancer, likely via various multiple mechanisms unique to each cancer type.

BCAA oxidation increases after feeding (Fig. 2). Conversely, briefly restricting food reduces oxidation of BCAAs, causing the elevation of their plasma levels. If fasting continues into starvation, BCAAs oxidation increases, providing gluconeogenic precursors to the liver. BCAAs oxidation rates fall again in severe starvation, presumably to conserve essential amino acids [77-79].

\section{CONCLUSION}

BCAAs are essential amino acids, and they are likely to be supplied to cancer cells from the tumor microenvironment or through protein degradation. They play prominent roles in tumor development and progression. The most well-defined mechanism of the BCAA and cancer association is the activation of the mTORC1 signalling. Also, BCAAs serve as building blocks in protein synthesis and are indirect sources of nitrogen supply for nucleotide (and non-essential amino acid) biosynthesis via the glutamate-glutamine axis. Aside from the mTOR pathway, other potential pathways within the framework of glucose and lipid metabolism might be a promising route in understanding the significance of BCAAs in cancer.

\section{FUTURE DIRECTIONS}

First, further epidemiological studies on the significance of dietary BCAAs in tumor development and progression in populations are needed to significantly improve our understanding of the role of dietary factors in cancer. Second, molecular studies exploring the profiles of interaction between dietary and intracellular plasma BCAAs in the context of tumor manifestation would be worthwhile. Third, whether BCAAs are potential epigenetic markers that can manipulate genetic predisposition to tumorigenesis is yet to be understood. Also, the differential roles of cytosolic BCAT1 and mitochondrial BCAT2 by cancer types cannot be underestimated in expanding our understanding of this topic.

\section{ACKNOWLEDGMENTS}

This review was registered in the International Prospective Register for Systematic Reviews (https://www.crd.york. ac.uk/prospero/display_record.php?ID=CRD42021243805). Also, APO is a Brain Pool Program Fellow of the National Research Foundation of Korea, funded by the Ministry of Science and ICT (2020H1D3A1A04081265).

\section{CONFLICTS OF INTEREST}

No potential conflicts of interest were disclosed.

\section{ORCID}

Min Kyu Jung, https://orcid.org/0000-0001-8749-408X

Akinkunmi Paul Okekunle, https://orcid.org/0000-0003-4825-4934

Jung Eun Lee, https://orcid.org/0000-0003-1141-878X

Mi Kyung Sung, https://orcid.org/0000-0002-3575-5628

Yun Jeong Lim, https://orcid.org/0000-0002-3279-332X

\section{REFERENCES}

1. DeBerardinis RJ, Chandel NS. Fundamentals of cancer metabolism. Sci Adv 2016;2:e1600200.

2. Martínez-Reyes I, Chandel NS. Cancer metabolism: looking forward. Nat Rev Cancer 2021;21:669-80.

3. Donaldson MS. Nutrition and cancer: a review of the evidence for an anti-cancer diet. Nutr J 2004;3:19.

4. Ravasco P. Nutrition in cancer patients. J Clin Med 2019;8:1211.

5. Bingham S, Riboli E. Diet and cancer--the European Prospective Investigation into Cancer and Nutrition. Nat Rev Cancer 2004;4:206-15.

6. Frank SA. Genetic predisposition to cancer - insights from population genetics. Nat Rev Genet 2004;5:764-72.

7. Ginsburg O, Ashton-Prolla P, Cantor A, Mariosa D, Brennan P. The role of genomics in global cancer prevention. Nat Rev Clin Oncol 2021;18:116-28.

8. Faubert B, Solmonson A, DeBerardinis RJ. Metabolic 
reprogramming and cancer progression. Science 2020;368: eaaw5473.

9. Wang TJ, Larson MG, Vasan RS, Cheng S, Rhee EP, McCabe $\mathrm{E}$, et al. Metabolite profiles and the risk of developing diabetes. Nat Med 2011;17:448-53.

10. Okekunle AP, Li Y, Liu L, Du S, Wu X, Chen Y, et al. Abnormal circulating amino acid profiles in multiple metabolic disorders. Diabetes Res Clin Pract 2017;132:45-58.

11. Zheng Y, Li Y, Qi Q, Hruby A, Manson JE, Willett WC, et al. Cumulative consumption of branched-chain amino acids and incidence of type 2 diabetes. Int J Epidemiol 2016;45:1482-92.

12. Nagata C, Nakamura K, Wada K, Tsuji M, Tamai Y, Kawachi T. Branched-chain amino acid intake and the risk of diabetes in a Japanese community: the Takayama study. Am J Epidemiol 2013;178:1226-32.

13. Okekunle AP, Zhang M, Wang Z, Onwuka JU, Wu X, Feng R, et al. Dietary branched-chain amino acids intake exhibited a different relationship with type 2 diabetes and obesity risk: a meta-analysis. Acta Diabetol 2019;56:187-95.

14. Harper AE, Miller RH, Block KP. Branched-chain amino acid metabolism. Annu Rev Nutr 1984;4:409-54.

15. Bolster DR, Jefferson LS, Kimball SR. Regulation of protein synthesis associated with skeletal muscle hypertrophy by insulin-, amino acid- and exercise-induced signalling. Proc Nutr Soc 2004;63:351-6.

16. Dill KA. Dominant forces in protein folding. Biochemistry 1990; 29:7133-55.

17. Brosnan JT, Brosnan ME. Branched-chain amino acids: enzyme and substrate regulation. J Nutr 2006;136(1 Suppl):207S-11S.

18. Amorim Franco TM, Blanchard JS. Bacterial branchedchain amino acid biosynthesis: structures, mechanisms, and drugability. Biochemistry 2017;56:5849-65.

19. McCourt JA, Duggleby RG. Acetohydroxyacid synthase and its role in the biosynthetic pathway for branched-chain amino acids. Amino Acids 2006;31:173-210.

20. Chou PY, Fasman GD. Empirical predictions of protein conformation. Annu Rev Biochem 1978;47:251-76.

21. Matsumoto T, Nakamura K, Matsumoto $H$, Sakai R, Kuwahara $\mathrm{T}$, Kadota $\mathrm{Y}$, et al. Bolus ingestion of individual branched-chain amino acids alters plasma amino acid profiles in young healthy men. Springerplus 2014;3:35.

22. Monirujjaman M, Ferdouse A. Metabolic and physiological roles of branched-chain amino acids. Adv Mol Biol 2014;2014:364976.

23. Neinast M, Murashige D, Arany Z. Branched chain amino acids. Annu Rev Physiol 2019;81:139-64.

24. Ichihara A, Koyama E. Transaminase of branched chain amino acids. I. Branched chain amino acids- $\alpha$-ketoglutarate transaminase. J Biochem 1966;59:160-9.

25. Wu G. Amino acids: metabolism, functions, and nutrition. Amino Acids 2009;37:1-17.

26. Wahren J, Felig P, Hagenfeldt L. Effect of protein ingestion on splanchnic and leg metabolism in normal man and in patients with diabetes mellitus. J Clin Invest 1976;57:987-99.

27. Louard RJ, Barrett EJ, Gelfand RA. Effect of infused branched- chain amino acids on muscle and whole-body amino acid metabolism in man. Clin Sci (Lond) 1990;79:457-66.

28. Everman S, Mandarino LJ, Carroll CC, Katsanos CS. Effects of acute exposure to increased plasma branched-chain amino acid concentrations on insulin-mediated plasma glucose turnover in healthy young subjects. PLoS One 2015;10:e0120049.

29. Shimomura Y, Kitaura Y, Kadota Y, Ishikawa T, Kondo Y, Xu M, et al. Novel physiological functions of branched-chain amino acids. J Nutr Sci Vitaminol (Tokyo) 2015;61 Suppl:S112-4.

30. Gannon NP, Schnuck JK, Vaughan RA. BCAA metabolism and insulin sensitivity - dysregulated by metabolic status? Mol Nutr Food Res 2018;62:e1700756.

31. Wolfe RR, Cifelli AM, Kostas G, Kim IY. Optimizing protein intake in adults: interpretation and application of the recommended dietary allowance compared with the Acceptable Macronutrient Distribution Range. Adv Nutr 2017;8:266-75.

32. Fulgoni VL 3rd. Current protein intake in America: analysis of the National Health and Nutrition Examination Survey, 2003-2004. Am J Clin Nutr 2008;87:1554S-7S.

33. Matthews DE, Motil KJ, Rohrbaugh DK, Burke JF, Young VR, Bier DM. Measurement of leucine metabolism in man from a primed, continuous infusion of L-[1-3C]leucine. Am J Physiol 1980;238:E473-9.

34. Wolfe RR, Goodenough RD, Wolfe MH, Royle GT, Nadel ER. Isotopic analysis of leucine and urea metabolism in exercising humans. J Appl Physiol Respir Environ Exerc Physiol 1982;52:458-66.

35. Short KR, Vittone JL, Bigelow ML, Proctor DN, Nair KS. Age and aerobic exercise training effects on whole body and muscle protein metabolism. Am J Physiol Endocrinol Metab 2004;286:E92-101.

36. Suryawan A, Orellana RA, Fiorotto ML, Davis TA. Triennial Growth Symposium: leucine acts as a nutrient signal to stimulate protein synthesis in neonatal pigs. J Anim Sci 2011;89:2004-16.

37. May ME, Buse MG. Effects of branched-chain amino acids on protein turnover. Diabetes Metab Rev 1989;5:227-45.

38. Anthony JC, Yoshizawa F, Anthony TG, Vary TC, Jefferson LS, Kimball SR. Leucine stimulates translation initiation in skeletal muscle of postabsorptive rats via a rapamycin-sensitive pathway. J Nutr 2000;130:2413-9.

39. Wang $X$, Proud CG. The mTOR pathway in the control of protein synthesis. Physiology (Bethesda) 2006;21:362-9.

40. Mayers JR, Wu C, Clish CB, Kraft P, Torrence ME, Fiske BP, et al. Elevation of circulating branched-chain amino acids is an early event in human pancreatic adenocarcinoma development. Nat Med 2014;20:1193-8.

41. Nezami Ranjbar MR, Luo Y, Di Poto $C$, Varghese RS, Ferrarini A, Zhang C, et al. GC-MS based plasma metabolomics for identification of candidate biomarkers for hepatocellular carcinoma in Egyptian cohort. PLoS One 2015;10:e0127299.

42. Budhathoki S, Iwasaki M, Yamaji T, Yamamoto $H$, Kato $Y$, Tsugane S. Association of plasma concentrations of branchedchain amino acids with risk of colorectal adenoma in a large Japanese population. Ann Oncol 2017;28:818-23. 
Jung et al.

43. Zeleznik OA, Balasubramanian R, Ren Y, Tobias DK, Rosner BA, Peng $C$, et al. Branched-chain amino acids and risk of breast cancer. JNCI Cancer Spectr 2021;5:pkab059.

44. Mayers JR, Torrence ME, Danai LV, Papagiannakopoulos T, Davidson SM, Bauer MR, et al. Tissue of origin dictates branched-chain amino acid metabolism in mutant Kras-driven cancers. Science 2016;353:1161-5.

45. Ananieva EA, Powell JD, Hutson SM. Leucine metabolism in $\mathrm{T}$ cell activation: mTOR signaling and beyond. Adv Nutr 2016;7:798S-805S

46. Wolfson RL, Chantranupong L, Saxton RA, Shen K, Scaria SM, Cantor JR, et al. Sestrin2 is a leucine sensor for the mTORC1 pathway. Science 2016;351:43-8.

47. Saxton RA, Knockenhauer KE, Wolfson RL, Chantranupong L, Pacold ME, Wang T, et al. Structural basis for leucine sensing by the Sestrin2-mTORC1 pathway. Science 2016;351:53-8.

48. Chang IW, Wu WJ, Wang YH, Wu TF, Liang PI, He HL, et al. BCAT1 overexpression is an indicator of poor prognosis in patients with urothelial carcinomas of the upper urinary tract and urinary bladder. Histopathology 2016;68:520-32.

49. Xu Y, Yu W, Yang T, Zhang M, Liang C, Cai X, et al. Overexpression of BCAT1 is a prognostic marker in gastric cancer. Hum Pathol 2018;75:41-6.

50. Zheng YH, Hu WJ, Chen BC, Grahn TH, Zhao YR, Bao HL, et al. BCAT1, a key prognostic predictor of hepatocellular carcinoma, promotes cell proliferation and induces chemoresistance to cisplatin. Liver Int 2016;36:1836-47.

51. Han S, Liu Y, Cai SJ, Qian M, Ding J, Larion M, et al. IDH mutation in glioma: molecular mechanisms and potential therapeutic targets. Br J Cancer 2020;122:1580-9.

52. Tönjes M, Barbus S, Park YJ, Wang W, Schlotter M, Lindroth AM, et al. BCAT1 promotes cell proliferation through amino acid catabolism in gliomas carrying wild-type IDH1. Nat Med 2013;19:901-8.

53. Xu W, Yang H, Liu Y, Yang $Y$, Wang $\mathrm{P}$, Kim SH, et al. Oncometabolite 2-hydroxyglutarate is a competitive inhibitor of $\alpha$-ketoglutarate-dependent dioxygenases. Cancer Cell 2011; 19:17-30.

54. Raffel S, Falcone M, Kneisel N, Hansson J, Wang W, Lutz C, et al. BCAT1 restricts $\alpha K G$ levels in AML stem cells leading to IDHmut-like DNA hypermethylation. Nature 2017;551:384-8.

55. McBrayer SK, Mayers JR, DiNatale GJ, Shi DD, Khanal $J$, Chakraborty AA, et al. Transaminase inhibition by 2-hydroxyglutarate impairs glutamate biosynthesis and redox homeostasis in glioma. Cell 2018;175:101-16.e25.

56. Sheikh TN, Patwardhan PP, Cremers S, Schwartz GK. Targeted inhibition of glutaminase as a potential new approach for the treatment of NF1 associated soft tissue malignancies. Oncotarget 2017;8:94054-94068.

57. Zhang L, Han J. Branched-chain amino acid transaminase 1 (BCAT1) promotes the growth of breast cancer cells through improving mTOR-mediated mitochondrial biogenesis and function. Biochem Biophys Res Commun 2017;486:224-31.

58. Sripathy S, Lee M, Vasioukhin V. Mammalian Llgl2 is necessary for proper branching morphogenesis during placental development. Mol Cell Biol 2011;31:2920-33.

59. Saito Y, Li L, Coyaud E, Luna A, Sander C, Raught B, et al. LLGL2 rescues nutrient stress by promoting leucine uptake in ER $^{+}$breast cancer. Nature 2019;569:275-9.

60. Tobias DK, Chai B, Tamimi RM, Manson JE, Hu FB, Willett WC, et al. Dietary intake of branched chain amino acids and breast cancer risk in the NHS and NHS II prospective cohorts. $\mathrm{JNCl}$ Cancer Spectr 2021;5:pkab032.

61. Qiu Y, Cai G, Su M, Chen T, Zheng X, Xu Y, et al. Serum metabolite profiling of human colorectal cancer using GCTOFMS and UPLC-QTOFMS. J Proteome Res 2009;8:4844-50.

62. Delphan M, Lin T, Liesenfeld DB, Nattenmüller J, Böhm JT, Gigic B, et al. Associations of branched-chain amino acids with parameters of energy balance and survival in colorectal cancer patients: results from the ColoCare study. Metabolomics 2018; 2018:22.

63. Cross AJ, Moore SC, Boca S, Huang WY, Xiong X, StolzenbergSolomon R, et al. A prospective study of serum metabolites and colorectal cancer risk. Cancer 2014;120:3049-57.

64. Tran TT, Medline A, Bruce WR. Insulin promotion of colon tumors in rats. Cancer Epidemiol Biomarkers Prev 1996;5:1013-5.

65. Björk J, Nilsson J, Hultcrantz R, Johansson C. Growth-regulatory effects of sensory neuropeptides, epidermal growth factor, insulin, and somatostatin on the non-transformed intestinal epithelial cell line IEC-6 and the colon cancer cell line HT 29. Scand J Gastroenterol 1993;28:879-84.

66. Corpet DE, Jacquinet C, Peiffer G, Taché S. Insulin injections promote the growth of aberrant crypt foci in the colon of rats. Nutr Cancer 1997;27:316-20.

67. Shimizu M, Shirakami Y, Iwasa J, Shiraki M, Yasuda Y, Hata K, et al. Supplementation with branched-chain amino acids inhibits azoxymethane-induced colonic preneoplastic lesions in male C57BL/KsJ-db/db mice. Clin Cancer Res 2009;15:3068-75.

68. Denkert C, Budczies J, Weichert W, Wohlgemuth G, Scholz M, Kind $\mathrm{T}$, et al. Metabolite profiling of human colon carcinoma-deregulation of TCA cycle and amino acid turnover. Mol Cancer 2008;7:72.

69. Murata K, Moriyama M. Isoleucine, an essential amino acid, prevents liver metastases of colon cancer by antiangiogenesis. Cancer Res 2007;67:3263-8.

70. Rossi M, Mascaretti F, Parpinel M, Serraino D, Crispo A, Celentano $\mathrm{E}$, et al. Dietary intake of branched-chain amino acids and colorectal cancer risk. Br J Nutr 2021;126:22-7.

71. Katagiri R, Song M, Zhang X, Lee DH, Tabung FK, Fuchs CS, et al. Dietary intake of branched-chain amino acids and risk of colorectal cancer. Cancer Prev Res (Phila) 2020;13:65-72.

72. Xue P, Zeng F, Duan Q, Xiao J, Liu L, Yuan P, et al. BCKDK of BCAA catabolism cross-talking with the MAPK pathway promotes tumorigenesis of colorectal cancer. EBioMedicine 2017;20:50-60.

73. Lee JH, Cho YR, Kim JH, Kim J, Nam HY, Kim SW, et al. Branched-chain amino acids sustain pancreatic cancer growth by regulating lipid metabolism. Exp Mol Med 2019;51:1-11. 
74. Hattori A, Tsunoda M, Konuma T, Kobayashi M, Nagy T, Glushka $\mathrm{J}$, et al. Cancer progression by reprogrammed BCAA metabolism in myeloid leukaemia. Nature 2017;545:500-4.

75. Oktyabri D, Ishimura A, Tange S, Terashima M, Suzuki T. DOT1L histone methyltransferase regulates the expression of BCAT1 and is involved in sphere formation and cell migration of breast cancer cell lines. Biochimie 2016;123:20-31.

76. Mayers JR, Vander Heiden MG. Nature and nurture: what determines tumor metabolic phenotypes? Cancer Res 2017;77:
3131-4

77. Holeček M. Why are branched-chain amino acids increased in starvation and diabetes? Nutrients 2020;12:3087.

78. Brody T. Protein. In: Brody T, ed. Nutritional Biochemistry. 2nd ed. San Diego, Academic Press, pp 421-89, 1999.

79. Holecek M, Sprongl L, Tilser I. Metabolism of branched-chain amino acids in starved rats: the role of hepatic tissue. Physiol Res 2001;50:25-33. 